\title{
Psychotropic prescribing in mental health services and primary care
}

\author{
L. Alexander ${ }^{1,2}$ and J. Lyne ${ }^{3,4, *}$ \\ ${ }^{1}$ Tallaght Adult Mental Health Services, Sheaf House, Tallaght, Dublin 24, Ireland \\ ${ }^{2}$ Department of Psychiatry, Trinity College Dublin, Dublin 2, Ireland \\ ${ }^{3}$ Royal College of Surgeons in Ireland, 123 St. Stephen's Green, Dublin 2, Ireland \\ ${ }^{4}$ Wicklow Mental Health Services, Newcastle Hospital, Greystones, Co. Wicklow, Ireland
}

This issue of the Irish Journal of Psychological Medicine takes a detailed exploration of psychotropic prescribing practices in mental health services as well as primary care in both Ireland and the United Kingdom. Recent studies have suggested that the use of psychotropic medication across these populations has become increasingly commonplace. Shevlin et al. (pp 32-38) used data linkage techniques to report rates of antidepressant prescribing in Northern Ireland of between $12 \%$ and 14\% each year from 2011 to 2015, while $24 \%$ had been prescribed antidepressants at some stage across the 5 -year period. The authors noted the relatively high prescribing prevalence and also report that indicators of socio-economic disadvantage, such as unemployment, social renting and poor general health, were associated with increased antidepressant use. In a separate study, Boyle et al. (pp 15-23) reported the prevalence of antidepressant prescribing in a nationally representative sample of 7403 adults residing in England in 2007 using a different study methodology. In their sample, $5.6 \%$ of adults reported current use of antidepressants. They also found that unemployment was associated with increased antidepressant prescribing, along with other variables such as female gender, speaking English as a first language and childhood sexual abuse.

In another study highlighting mental illness and prescribing rates in Irish General Practices, O'Doherty et al. (pp 24-31) found that $16 \%$ of patients attending primary care in the previous 2 years (2011-2013) were reported to have psychological disorders, with depression and stress/anxiety being the commonest conditions. Among these patients, $34 \%$ received a psychological intervention while the majority $(81 \%)$ were treated with a pharmacological intervention. Antidepressants (58\%), benzodiazepines $(30 \%)$ and

*Address for correspondence: Dr. John Lyne, Wicklow Mental Health Services, Newcastle Hospital, Greystones, Co. Wicklow, Ireland.

(Email: johnpaul.lyne@hse.ie) antipsychotics (9\%) were the most commonly prescribed medications.

These three studies highlight the reliance on medication prescribing for managing mental health difficulties in Ireland and the United Kingdom, with much of this care likely provided in primary care. In a related review article, Tobin et al. (pp 59-66) examined possible reasons for variation in primary care prescribing. From the studies they reviewed, which were mostly based in the United Kingdom, increased antidepressant prescribing was found among populations with social deprivation and white ethnicity. General Practitioner preferences and patient characteristics, such as female gender and increased age, were also associated with higher antidepressant prescribing; however, there was not consensus among the studies examined on what factors might contribute most to higher psychotropic prescribing in General Practice.

The above studies provide a nice overview of psychotropic prescribing across populations and in primary care. In another very interesting study for all prescribing psychiatrists, Rowntree et al. (pp 8-14) move the focus on prescribing patterns to an Irish community mental health service. Over a 12-year period (2005-2016), there was a notable reduction in sedative prescribing, along with other more moderate reductions in antidepressant and mood stabiliser prescriptions. In a further examination of prescribing in Irish mental health services, Cleary et al. (pp 43-47) examined prescriptions issued to patients at the point of discharge from an acute psychiatric unit, a period recognised for increased suicide risk. The study suggests that changes in prescribing practices are warranted, after finding that $39 \%$ of discharge prescriptions contained potentially lethal doses of psychotropic medication.

Two articles in this issue discuss how best to organise community mental health teams. Khan et al. (pp 39-42) compared the continuity model of consultant care with the split model, where different consultants manage inpatient and outpatient care. Vaughan 
et al. (pp 48-54) also explored this issue as part of their wider discussion around the benefits of the sectorisation model for community mental health teams. In both studies, the majority of service users expressed a preference for continuous care rather than a separate specialised inpatient consultant, with maintenance of the therapeutic relationship identified as one of the primary reasons. Vaughan et al. also explored attitudes towards the sectorisation model for delivery of mental health services and nicely outline the advantages and disadvantages relating to different service delivery models.

In a correspondence, Hayward (pp 69-70) considered the 'wellness' movement and its impact on delivery of psychiatric treatments. Far removed from its clandestine origins based in asylums patients today are not only seeking treatments for mental health difficulties, but are also looking to play an active role in the delivery of services. This is a welcome development for service progression and can improve advocacy for increasing resources in mental health services. Hayward outlines that sometimes the wellness era movement can portray psychiatry as a field requiring substantial change, and the correspondence continues with a thought-provoking consideration of the pros and potential cons of the wellness movement. The correspondence also provides further narrative about how mental healthcare might be funded and delivered in the new landscape, including the option of encouraging people to seek support outside of a clinical setting.

Finally in a moving tribute to Dr Seamus Mac Suibhne (pp 3-7), Kelly and O'Loughlin describe a psychiatrist who embraced many aspects of psychiatry from philosophy to the colourful evolution of psychiatry, as well as the key importance of developing medical education. Seamus viewed psychiatry through a unique lens, one that incorporated its myriad influences, and he firmly placed compassion at the centre of clinical care. He was a dedicated family man and a highly valued colleague to many in his profession. In his appreciation of the broader context he was ahead of his time, and his influence as an inspiring psychiatrist will be felt long after his untimely passing.

\section{Conflict of interest}

The authors have no conflicts of interest to disclose. 